\title{
COMISSÃO ORGANIZADORA
}

\author{
Prof. Dr. João Santana da Silva \\ Coordenador do Programa de Pós-graduação em Imunologia Básica e A plicada \\ Prof. Dr. E duardo Antonio Donadi \\ Vice-Cordenador do Programa de Pós-graduação em I munologia Básica e A plicada \\ Profa. Dra Beatriz Rossetti Ferreira \\ Coordenadora da Comissão O rganizadora \\ Ana Cristine S. Ferreira \\ Secretária da Pós-graduação em I munologia Básica e A plicada
}

- Comissão Científica

Prof. D r. Ademilson Panunto Castelo

D ra. Alexandra I vo de Medeiros

D ra. A na O lívia de Souza

Profa. D ra. Beatriz Rossetti Ferreira

Dr. Victor Aquino

\section{- Comissão E ditorial}

Carlos Rodrigo Zárate Bladés

M irela de Barros Tamarozzi

Sandro G omes Soares

- Comissão de Marketing

A na Paula M oreira

$D$ aniela D antas M oré

$K$ aren Angélica Cavassani

- Comissão Social

Pryscilla Fanini Wowk

Renata Sesti Costa

Viviane Casagrande M ariguela

- Comissão Financeira

$K$ arina Alves de Toledo

Leandro Licursi de O liveira

Luciana Pereira Ruas

M arina O liveira e Paula

\section{PALESTRAN TES CON VIDADOS}

Dr. Paul J. Travers

Anthony N olan Research Institute-UK

Profa. Dra. Aldina Barral

FIOCRUZ - BA

Prof. Dr. Momtchilo Russo

ICB - USP

Profa. Dra. Angela K. Cruz

Facul dade de Medicina de Ribeirão Preto - USP

Prof. Dr. J orge Kalil

INCOR - USP 